\title{
Dechlorination of Trichloroethylene in Groundwater by Nanoscale Bimetallic Fe/Pd Particles
}

\author{
Tielong LI ${ }^{1,2}$, Shujing LI ${ }^{1}$, Yongchao LI ${ }^{1}$, Zhaohui JIN ${ }^{1}$ \\ ${ }^{1}$ College of Environmental Science and Engineering, Nankai University, Tianjin, China \\ ${ }^{2}$ Tianjin Key Laboratory of Environmental Remediation and Pollution Control/Ministry of Education Key Laboratory of \\ Environmental Processes and Criteria, Nankai University, Tianjin, China \\ E-mail: litielong@nankai.edu.cn \\ Received November 29, 2008; revised January 13, 2009; accepted February 14, 2009
}

\begin{abstract}
Palladium/iron bimetallic nanoparticles were synthesized using microemulsion method in the water-in-oil (W/O) microemulsion system, which was made up of iso-octane, cetyltrimethyl-ammonium bromide (CTAB), butanol and water and characterized by measuring the conductivity of the solution. Transmission electron microscope (TEM) and energy dispersive X-ray microanalysis (EDX) analysis showed that the average diameter of synthesized palladium/iron bimetallic nanoparticles was less than $80 \mathrm{~nm}$, which was much smaller than the particles produced by the solution method. The palladium/iron bimetallic nanoscale particles produced in the laboratory showed better performance on dechlorinating TCE than the other materials. The nanoscale $\mathrm{Fe} / \mathrm{Pd}$ particles exhibited high reactivity. When Pd content is $0.5 \%$, the best TCE dechlorination efficiency is achieved within 30min. And Fe/Pd nanoparticles show persistent reaction activity in some sense.
\end{abstract}

Keywords: Trichloroethylene, Microemulsion, Nanoscale Pd/Fe Particles

\section{Introduction}

Chlorinated hydrocarbons are dense non-aqueous phase liquids (DNAPLs), which are utilized by a number of industries as solvents in large quantities on a regular basis. Given this high frequency of use, handling, and transportation, along with past disposal and storage practices, DNAPL compounds presently represent a significant threat to soil and groundwater resources. Trichloroethylene (TCE), has been widely detected in areas adjacent to dry cleaners, automobile manufacturers or shops, asphalt processing plants, and military bases, listed as a priority pollutant by the US EPA, is one of the most commonly detected chlorinated organic compounds in surface water, groundwater and soil. Cleanup of soils and groundwater contaminated by chlorinated hydrocarbons such as TCE and PCBs has been a challenging task for decades.

Remediation of DNAPL-contaminated sites is especially critical because DNAPLs in the subsurface represent a long-term source for groundwater contamination.
Aquifers contaminated with DNAPLs are extremely difficult to remediate with standard pump-and-treat methods. Since DNAPLs are extremely difficult and costly to remove, a cost-effective, reliable technology is needed to treat DNAPL contaminants.

Various technologies have been explored for dechlorination of TCE, including bioremediation [1], thermal treatment [2], and permeable reactive barriers [3]. Among many technologies tested so far, abiotic dechlorination using zerovalent iron, $\mathrm{Fe}^{0}$, particles appears to be one of the most promising technologies [4,5]. The most common metal being utilized for this purpose is iron due to its dehalogenation efficiency, cost, and benign environmental impact. However, due to the limited reactivity, the TCE reduction rate of granular iron particles has been found very slow, with half-lives in the order of days or longer [6,7]. As a result, toxic intermediate byproducts such as vinyl chloride (VC) are often detected $[8,9]$. In order to further enhance the dechlorination reaction rates and minimize byproduct formation, bi-metal systems of palladized iron (Fe complex) were 
developed and used [10-12]. Various strategies have been explored to enhance the dechlorination rates using $\mathrm{Fe}^{0}$-based particles. Because dechlorination of chlorinated compounds by $\mathrm{Fe}^{0}$-based particles is a surface-mediated process, increasing the surface area of iron will increase the dechlorination rate. Therefore, reducing particle size can greatly enhance the degradation rate. Coating iron particles with a second catalytic metal such as $\mathrm{Pd}, \mathrm{Pt}, \mathrm{Ag}$, or Ni can also accelerate the dechlorination process and thereby prevent formation of toxic byproducts [13-15]. Zhang et al. reported that reducing Pd-coated iron particle size from millimeters to nanometers $(10-100 \mathrm{~nm})$ increased TCE degradation rate by 10-100 times. Typically, $\mathrm{Fe}^{0}$-based nanoparticles were prepared by reducing Fe (II) or Fe(III) in aqueous solution using a strong reducing agent (e.g., sodium borohydride, $\mathrm{NaBH}_{4}$ ). However, due to the extremely high reactivity, the initially formed nanoparticles tend to either react rapidly with surrounding media (e.g., dissolved oxygen (DO) or water) or agglomerate rapidly, resulting in the formation of much larger (in the micrometer to millimeter scale) particles or flocs and rapid loss in reactivity [16]. To overcome these drawbacks, a microemulsion $[17,18]$ with the cetyltrimethyl-ammonium bromide (CTAB) as the surfactant was used in the preparation of the $\mathrm{Fe} / \mathrm{Pd}$ nanoparticles and the chemical reactivity for degradation of TCE in water was studied in the experiment of dechlorination in this study. And the nanoparticles were characterized by using the transmission electron microscope (TEM) and energy dispersive X-ray microanalysis (EDX). The water-in-oil (W/O) microemulsion system was made up of iso-octane, CTAB, butanol and water, and was characterized by measuring the conductivity of the solution. The average diameter of synthesized palladium/iron bimetallic nanoparticles using the microemulsion method was less than $80 \mathrm{~nm}$, which was much smaller than the particles produced by the solution method. And then the primary objective of this work was to 1) prepare the nanoparticles in the CTAB microemulsion under inert conditions; and 2) characterize the resultant nanoparticles with respect to their physical stability and chemical reactivity for degradation of TCE in water. And the performance of the nanoparticles produced in the laboratory was studied by dechlorinating TCE. The degradation rate of TCE by the nano palladium/iron bimetallic particles produced in the laboratory was quantified and compared with nano iron particles.

\section{Experiments}

The experiments were divided into five parts. And laboratory grade chemicals were used in this experimental study.

\subsection{Conductivity}

The conductivity was measured using a microprocessor conductometer (DDS-307, ShangHai Precision and Scientific Instrument Corporation). The conductivity was measured to an accuracy of $\pm 0.5 \%$ of full scale reading within each range.

\subsection{Titration}

In order to construct the phase diagram of water/CTAB $+n$-butanol/iso-octane system, the titration method was used. Based on the literature review and preliminary tests, the mass ratio of co-surfactant-to-surfactant was fixed, and a predetermined amount of hydrocarbon iso-octane was added to the surfactant mixture. Water was then titrated into the octane-surfactant mixture (octane/(octane+surfactant) weight ratio of (S)) and changes were observed by visual inspection and conductivity measurement and the information was used in developing a phase diagram.

\subsection{Production of Fe/Pd Nanoparticles}

The preparation of iron nanoparticles was achieved by mixing rapidly the same volumes of two $\mathrm{W} / \mathrm{O}$ microemulsion solutions, with $\mathrm{FeSO}_{4}$ solubilized in one solution and $\mathrm{NaBH}_{4}$ as the reducing agent in the other solution. Then firstly, two water/CTAB/isooctane microemulsions ( $\mathrm{A}$ and $\mathrm{B}$ ), differing only in the type of aqueous phase, were prepared. The CTAB was selected as surfactant, with n-butanol as the co-surfactant and iso-octane as the oil phase. A solution of $0.2 \mathrm{M} \mathrm{FeSO}_{4}$ was added to the above mixture. The compositions of the microemulsion system used are summarized in Table 1. The weight ratio of iso-octane to surfactant was 3 . After completely stirring, a transparent yellow solution microemulsion A was obtained. With the same method microemulsion $\mathrm{B}$ was prepared, whereas the aqueous phase of microemulsion B was $1.3 \mathrm{~mol} / \mathrm{L} \mathrm{KBH}_{4}$ solution.

And then microemulsions $\mathrm{A}$ and $\mathrm{B}$ were quickly mixed at the protection of argon in a conical flask for preparation of nanoiron. The reduction reaction made the solution turbid with gas production and with a black color solid dispersed in the solution. The reduction reaction could be expressed as

$$
\mathrm{Fe}^{2+}+2 \mathrm{BH}_{4}^{-}+6 \mathrm{H}_{2} \mathrm{O} \rightarrow \mathrm{Fe}+2 \mathrm{~B}(\mathrm{OH})_{3}+7 \mathrm{H}_{2} \uparrow
$$

After the gas evolution, the mixtures were stirred for another $20 \mathrm{~min}$ with vigorous stirring $(3000 \mathrm{r} / \mathrm{min})$. And then loading of $\mathrm{Pd}$ to the $\mathrm{Fe}^{0}$ particles was accomplished by adding known quantities of $\mathrm{PdCl}_{2}$ into the microemulsion- $\mathrm{Fe}^{0}$ solution and allowing for reaction for 30 min under vacuum. The amount of Pd added in this study 
Table 1. Compositions of microemulsions system.

\begin{tabular}{ccccc}
\hline & Surfactant & Co-Surfactant & Oil & Aqueous \\
\hline ME & CTAB & butanol & iso-octane & $\mathrm{FeSO}_{4}$ \\
Weight (\%) & 11 & 10 & 31 & 48 \\
\hline
\end{tabular}

was $0.5 \%(\mathrm{w} / \mathrm{w})$ of Fe. Palladium was deposited on the iron surface through the following redox reaction 2 ,

$$
\mathrm{Pd}^{2+}+2 \mathrm{Fe} \rightarrow \mathrm{Pd}+\mathrm{Fe}^{2+}
$$

At the end of the reaction, the microemulsion system with the well-dispersed iron nanoparticles was transferred into a sealed vessel filled with argon by a double-tipped needle. The resulting black-gray solids were settled by magnet, and the supernatant was decanted. Then the solids were washed with deionized water and finally with a mixture of anhydrous ethanol and acetone (volume ratio is 1:1) for six times, respectively. Finally, the resultant black-gray solids were dried under argon atomosphere, and then stored in another sealed vessel filled with argon.

\subsection{Characterization of Particles}

The particles obtained using the microemulsion were characterized using the Philips EM400ST transmission electron microscope (TEM) and energy dispersive X-ray microanalysis (EDX).

\subsection{Dechlorination of TCE}

Pd content can affect TCE dechlorination efficiency strongly. In order to select the optimal $\mathrm{Fe} / \mathrm{Pd}$ radio, experiments were designed to investigate the effect of $\mathrm{Pd}$ content on TCE dechlorination efficiency of nanoscale $\mathrm{Pd} / \mathrm{Fe}$ within $30 \mathrm{~min}$. In this study, $\mathrm{Pd} / \mathrm{Fe}$ ratios were $0.2 \%, 0.5 \%, 1 \%, 2 \%$ respectively. For comparison, $\mathrm{Fe}$ and $\mathrm{Fe} / \mathrm{Pd}$ nanoparticles were also prepared following similar procedures but use the liquid reduction method. Experiments were conducted to investigate the reduction of TCE using the two synthesized iron particles. Dechlorination of $15 \mathrm{mg} / \mathrm{L}$ TCE solution with the $1.5 \mathrm{~g} / \mathrm{L}$ iron-to-solution loading was investigated. TCE solutions $(173 \mathrm{~mL})$ were mixed with the iron particles in separate bottles with Teflon coated caps. The mix was stirred using a platform shaker operated at $200 \mathrm{rpm}$. The concentration of TCE was analyzed using a HP 6890 GC equipped with an electron capture detector (ECD).

Experiments were also designed to evaluate $\mathrm{Fe} / \mathrm{Pd}$ nanoparticles' persistent activity. Batch experiments were conducted in $175 \mathrm{ml}$ serum bottles. In each batch bottle, $45 \mu \mathrm{L}$ TCE stock solution was repeatedly spiked into $173 \mathrm{ml}$ deionized water which was deoxygenized with Argon. Initial TCE concentration is $56 \mathrm{~g} / \mathrm{L}$. The solution contained $0.2768 \mathrm{~g}$ of $\mathrm{Fe} / \mathrm{Pd}$ nanoparticles.

\section{Results and Discussion}

\subsection{Phase Behavior of Water/Mixed-Surfactants/ Isooctane Microemulsion System}

In order to construct the phase diagram of water/mixedsurfactants/iso-octane microemulsion system, the titration method was used. The optimum weight ratio mentioned above was used to prepare the mixed-surfactants/ n-butanol mixture (EM). A predetermined amount of hydrocarbon iso-octane was added to the surfactant mixture. Water was then titrated into the iso-octane/surfactant mixture and changes were observed by visual inspection and conductivity measurement and the information was used to develop a phase diagram. The phase behavior of the water/mixed-surfactants/iso-octane system is represented in Figure 1. As can be seen in Figure 1, an ideal $\mathrm{W} / \mathrm{O}$ region has been observed where n-butanol: mixedsurfactants $=1: 2$ (weight ratio), which will be used as the microemulsion system to produce nanoscale particles.

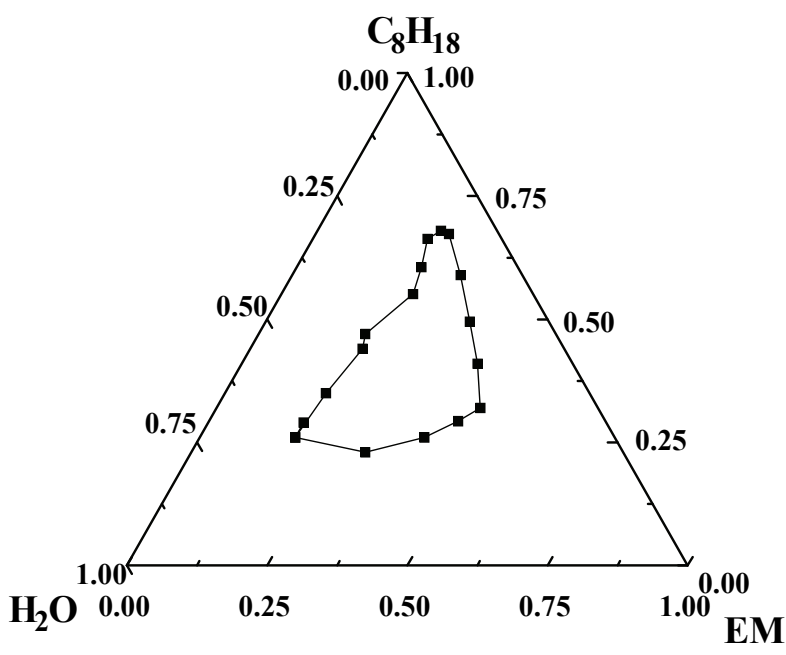

Figure 1. Microemulsion phase diagram of CTAB/Butanol/ Octane/ $\mathrm{H}_{2} \mathrm{O}$ system. 


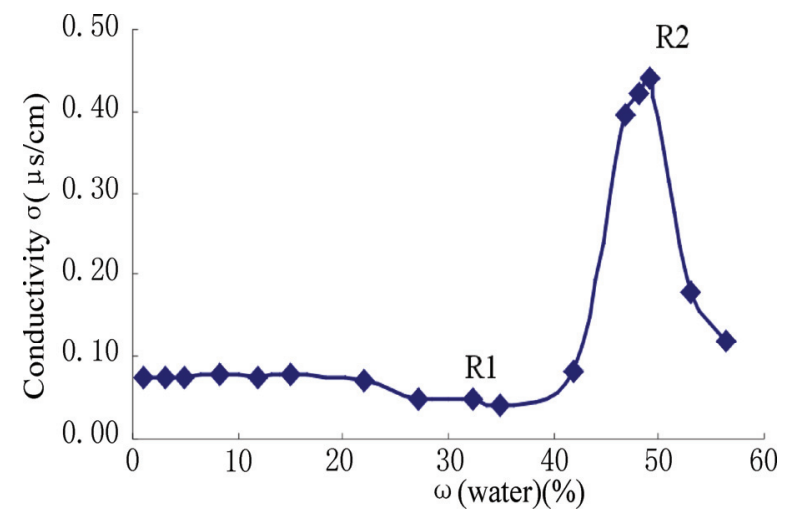

Figure 2. Variation of conductivity with amount of water in microemulsion.
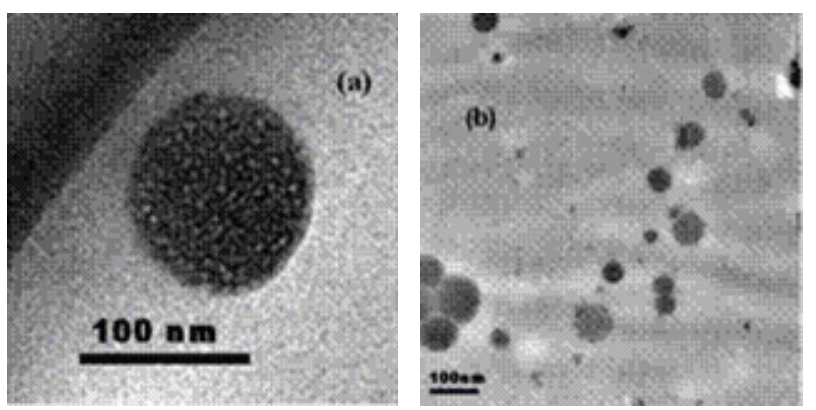

Figure 3. Characterizing the nanoscale Fe/Pd particles produced by microemulsion method; (a), (b) transmission electron micrograph (TEM) of the particles.

\subsection{Conductivity}

The variation of conductivity with different water con- tent (W0) is shown in Figure 2. Two turning points $(\mathrm{R} 1, \mathrm{R} 2)$ are found in Figure 2. Almost no change was observed in the initial conductivity of the iso-octane/surfactant mixture before R1. This is attributed to the formation of surfactant-bonded water in the system. But after R1 the conductivity increased sharply upon addition of the water into the iso-octane/surfactant mixture since it formed water-in-oil (W/O) microemulsion. In this region, water was present in micelle form and the water droplet size and number increased with an increased amount of water. When the droplet size increased, it was easy to exchange charges between the droplets and hence the conductivity increased. The conductivity reached a maximum when the water content was $50 \%$ (wt\%, R2). This can be explained by the saturation of droplets and the percolation of charges through the droplet clusters with minimum resistance. When more water was added to the system after R2, it leads to phase separation and the conductivity was lowered. The optically clear $\mathrm{W} / \mathrm{O}$ microemulsion system which was formed between R1 and R2 can be used as microreactors for preparation of nanoscale materials.

\subsection{Characterization of Synthesized Nanoscale Particles}

A small amount of microemulsion containing the palladium/iron bimetallic particles were withdrawn from the reaction vessel, and then preserved in a small sealed glass tube. For the TEM and EDX investigations, a small drop of microemulsion containing iron nanoparticles was deposited on a copper grid.

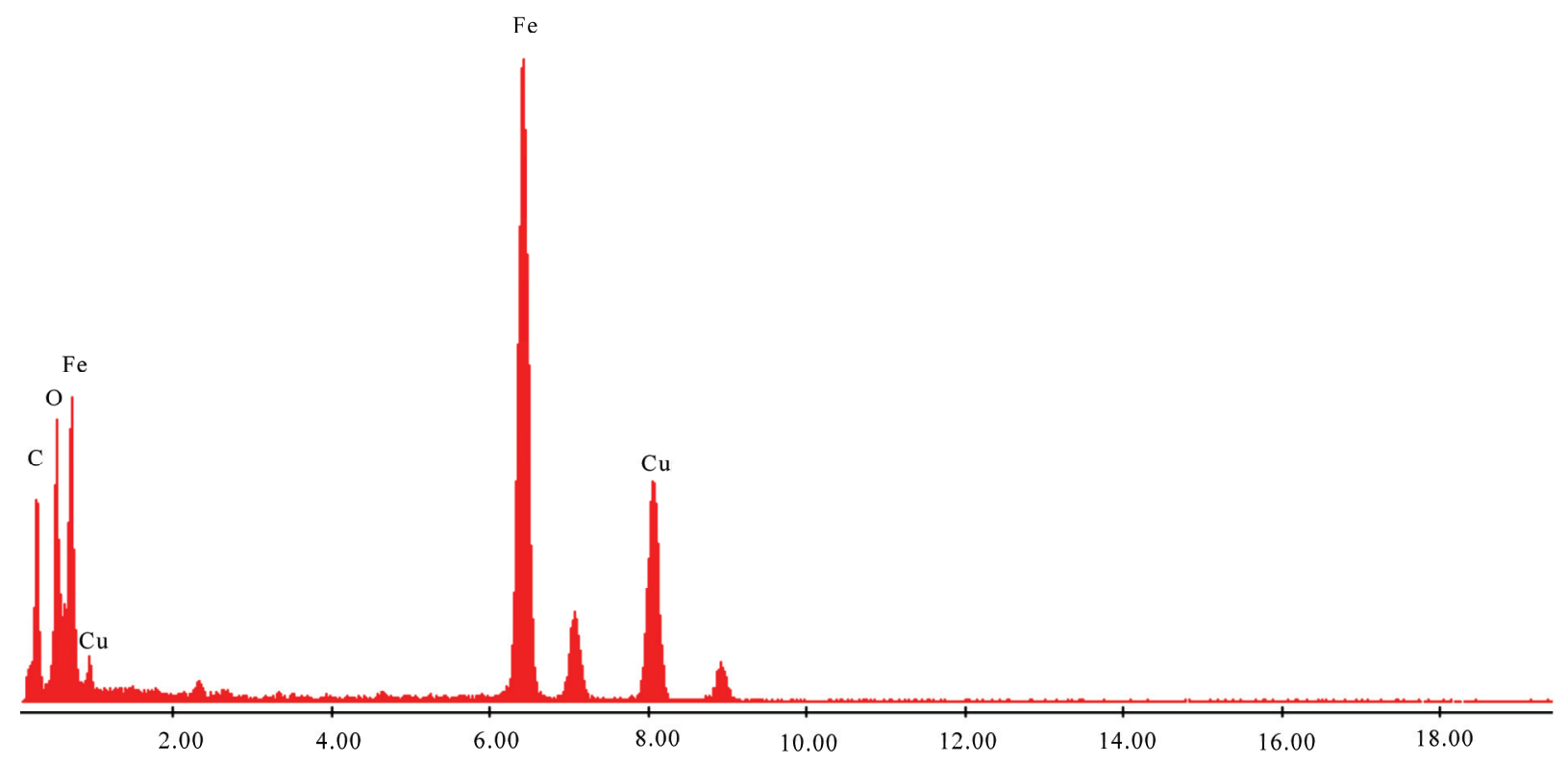

Figure 4. Energy dispersive X-ray microanalysis (EDX) of products. 


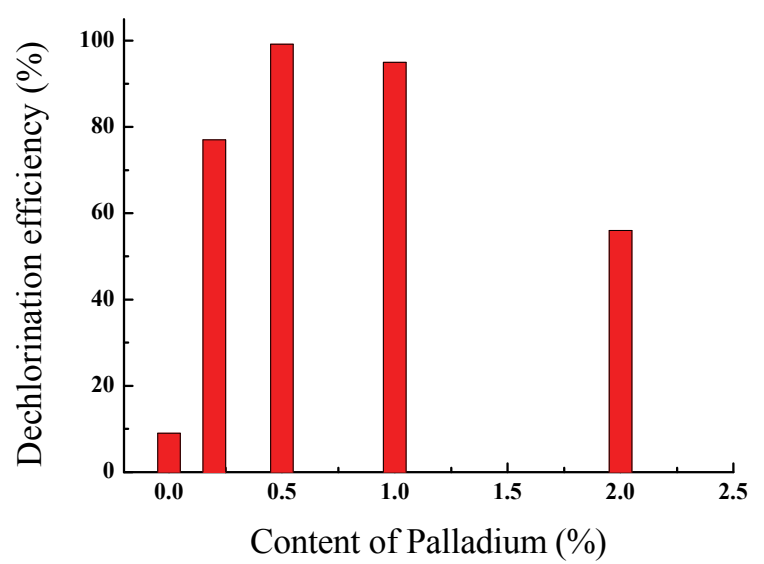

Figure 5. Effect of Pd content on TCE dechlorination efficiency of nanoscale $\mathrm{Pd} / \mathrm{Fe}$ (Reaction conditions: catalyst added $1.6 \mathrm{~g} / \mathrm{L}, \mathrm{T}=25^{\circ} \mathrm{C}, \mathrm{pH}=6.98, \mathrm{TCE}$ concentration $=14.8$ $\mathrm{mg} / \mathrm{L}$, stirring at $250 \mathrm{rpm}$, reaction for $30 \mathrm{~min}$ ).

Morphology of the particles is shown in Figure 3(a), (b). From the TEM images, the particles were nearly spherical in shape and uniform in size with the average particle size less than $80 \mathrm{~nm}$. The EDX for the sample is shown in Figure 4. It also shows that the product consisted of $\mathrm{Fe}$ and $\mathrm{Pd}$, and no other notable peaks were observed.

\subsection{Dechlorination of TCE}

As shown in Figure 5, it is apparent that Pd content affects TCE dechlorination efficiency of nanoscale $\mathrm{Pd} / \mathrm{Fe}$. By comparison, Fe nanoparticles with Pd showed significant dechlorination. When Pd content was $0.5 \%$, the best TCE dechlorination efficiency was achieved. But, Lower TCE was observed along with Pd content increasing. When Pd content was 2\%, TCE dechlorination efficiency was merely $60 \%$. Dechlorination efficiency decreasing was probably attributable to Pd films coating on the surface of Fe nanoparticles closely, that prevented Fe oxidation.

Figure 6 shows that degradation of TCE can be greatly enhanced when a small fraction $(0.5 \%$ of $\mathrm{Fe})$ of $\mathrm{Pd}$ was coated on the Fe particles. At the rather modest Fe dose of $1.5 \mathrm{~g} / \mathrm{L}$, even the NPI particles prepared by liquid method were able to eliminate $61 \%$ of TCE in the batch reactor within $0.5 \mathrm{~h}$. When the particles were prepared by microemulsion, $98 \%$ of TCE was destroyed within $0.5 \mathrm{~h}$. In contrast, a TCE degradation of only $8 \%$ was observed when the nanoscale Fe particles were prepared by microemulsion.

It can conclude that dechlorination efficiency of trichloroethylene with $\mathrm{Fe} / \mathrm{Pd}$ nanoparticles is above $99 \%$ in $30 \mathrm{~min}$ from Figure 7. It is also expected that the nanoparticles can remain reactive for extended period of time in the subsurface environment.

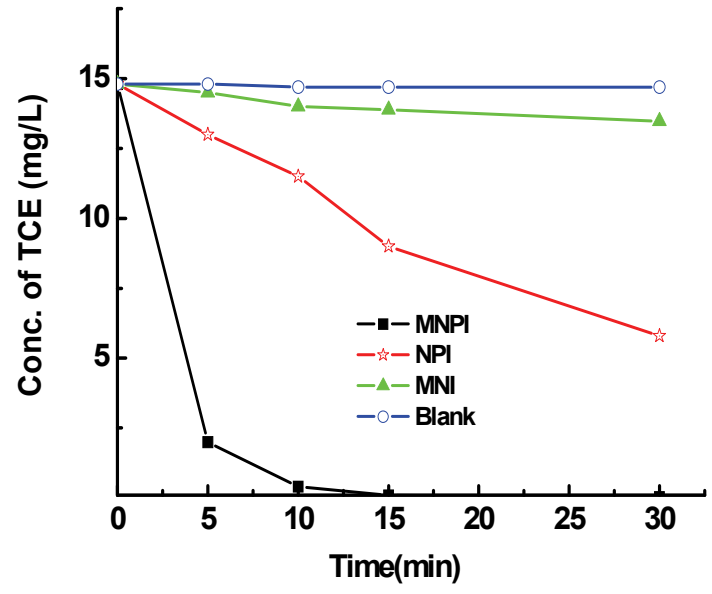

Figure 6. Dechlorination of TCE using nanoscale iron particles (MNI), nanoscale Fe/Pd particles (MNPI) prepared in microemulsion method, and nanoscale $\mathrm{Fe} / \mathrm{Pd}$ particles prepared in liquid method (NPI). Fe dose was $1.5 \mathrm{~g} / \mathrm{L}$ in all cases. Pd-to-iron ratio was $0.5 / 100(w / w)$.

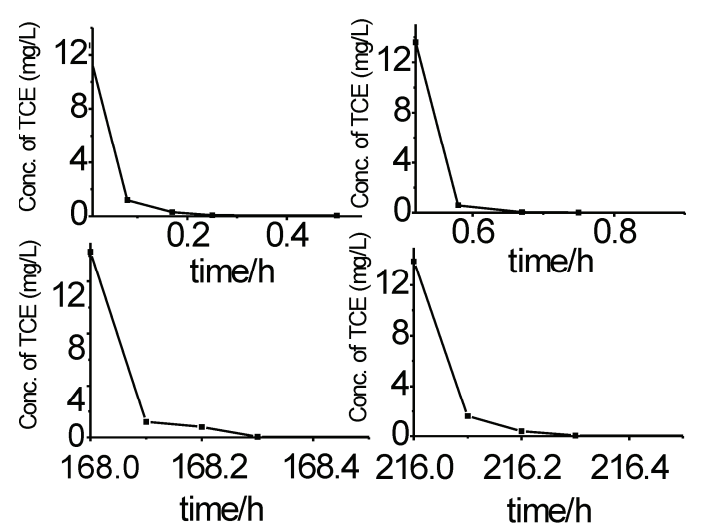

Figure 7. TCE concentration change with time in a multiple spiking experiment (reaction conditions: $\mathrm{Fe} / \mathrm{Pd}$ added $\mathbf{1 . 6}$ $\mathrm{g} / \mathrm{L}, \mathrm{T}=25^{\circ} \mathrm{C}, \mathrm{pH}=6.98$, $\mathrm{TCE}$ concentration $=14.8 \mathrm{mg} / \mathrm{L}$, stirring at 250rpm)

\section{Conclusions}

Based on the experimental study, the following conclusions are advanced:

Iron and $\mathrm{Fe} / \mathrm{Pd}$ nanoparticles were successfully produced by the solution method and the microemulsion method. For the microemulsion method, the particles were uniform in size and were less than $80 \mathrm{~nm}$, and appear to be clearly discrete and well-dispersed.

It showed in our results that the $\mathrm{Fe} / \mathrm{Pd}$ nanoparticles synthesized in our lab have the potential of remediation of TCE. The reduction rate of TCE by the microemulsion $\mathrm{Fe} / \mathrm{Pd}$ product was the highest compared with the solution product and to the iron prepared by microemulsion. Particle size is an important factor in determining the degradation rate of TCE. 
The nanoscale $\mathrm{Fe} / \mathrm{Pd}$ particles exhibited high reactivity. When Pd content is $0.5 \%$, the best TCE dechlorination efficiency is achieved. And Fe/Pd nanoparticles show persistent reaction activity in some sense.

\section{Acknowledgments}

This work was supported by Doctoral Fund for Young Scholar of Ministry of Education of China (No. 20070055053).

\section{References}

[1] J. MunakataMarr, P. L. McCarty, and M. S. Shields, "Enhancement of trichloroethylene degradation in aquifer microcosms bioaugmented with wild type and genetically altered Burkholderia (Pseudomonas) cepacia G4 and PR1," Environmental Science and Technology, Vol. 30, No. 6, pp. 2045-2052, 1996.

[2] A. K. Friis, H. J. Albrechtsen, and G. Heron, "Redox processes and release of organic matter after thermal treatment of a TCE-Contaminated aquifer," Environmental Science and Technology, Vol. 39, No. 15, pp. 5787-5795, 2005.

[3] H. Shen and J. T. Wilson, "Trichloroethylene removal from groundwater in flow-through columns simulating a permeable reactive barrier constructed with plant mulch," Environmental Science and Technology, Vol. 41, No. 11, pp. 4077-4083, 2007.

[4] L. J. Matheson and P. G. Tratnyek, "Reductive dehalogenation of chlorinated methanes by iron metal," Environmental Science and Technology, Vol. 28, No. 12, pp. 2045-2053, 1994.

[5] C. B. Wang and W. X. Zhang, "Synthesizing nanoscale iron particles for rapid and complete dechlorination of TCE and PCBs." Environmental Science and Technology, Vol. 31, No. 7, pp. 2154-2156, 1997.

[6] T. L. Johnson, M. M. Scherer, and P. G. Tratnyek, "Kinetics of halogenated organic compound degradation by iron metal," Environmental Science and Technology, Vol. 30, No. 8, pp. 2634-2640, 1996.

[7] G. D. Sayles, G. You, M. Wang, and M. J. Kupferle, "DDT, DDD, and DDE dechlorination by zerovalent iron," Environmental Science and Technology, Vol. 31, No. 12, pp. 3448-3454, 1997.
[8] W. A. Arnold and A. L. Roberts, "Pathways and kinetics of chlorinated ethylene and chlorinated acetylene reaction with $\mathrm{Fe}(0)$ particles," Environmental Science and Technology, Vol. 34, No. 9, pp. 1794-1805, 2000.

[9] W. S. Orth and R. W. Gillham, "Dechlorination of trichloroethene in aqueous solution using $\mathrm{Fe}^{0}$," Environmental Science and Technology, Vol. 30, No. 1, pp. 66-71, 1996.

[10] T. Li and J. Farrell, "Reductive dechlorination of trichloroethene and carbon tetrachloride using iron and palladized-iron cathodes," Environmental Science and Technology, Vol. 34, No. 1, pp. 173-179, 2000.

[11] P. Zhang, X. Tao, Z. Li, and R. S. Bowman, ,"Enhanced perchloroethylene reduction in column systems using surfactant-modified zeolite/zero-valent iron pellets," Environmental Science and Technology, Vol. 36, No. 16, pp. 3597-3603, 2002.

[12] G. V. Lowry and M. Reinhard, "Pd-catalyzed TCE dechlorination in groundwater: solute effects, biological control, and oxidative catalyst regeneration," Environmental Science and Technology, Vol. 34, No. 15, pp. 3217-3223, 2000.

[13] W. X. Zhang, C. B. Wang, and H. L. Lien, "Treatment of chlorinated organic contaminants with nanoscale bimetallic particles," Catalogue Today, Vol. 40, No. 4, pp. 387-395, 1998.

[14] Y. Xu and W. X. Zhang, "Subcolloidal Fe/Ag particles for reductive dehalogenation of chlorinated benzenes," Industrial And Engineering Chemistry Research, Vol. 39, No. 7, pp. 2238-2244, 2000.

[15] B. Schrick, J. L. Blough, A. D. Jones, and T. E. Mallouk, "Hydrodechlorination of trichloroethylene to hydrocarbons using bimetallic nickel-iron nanoparticles," Chemistry of Materials, Vol. 14, No. 12, pp. 5140-5147, 2002.

[16] F. He and D. Y. Zhao, "Preparation and characterization of a new class of starch-stabilized bimetallic nanoparticles for degradation of chlorinated hydrocarbons in water," Environmental Science and Technology, Vol. 39, No. 9, pp. 3314-3320, 2005.

[17] F. Li, C. Vipulanandan, and K. K. Mohanty, "Microemulsion and solution approaches to nanoparticle iron production of degradation of trichloroethylene," Colloids Surface, Vol. 223, No. 1-3, pp. 103-112, 2003.

[18] A. J. Zarur and J. Y. Ying, "Reverse microemulsion synthesis of nanostructured complex oxides for catalytic combustion," Nature, Vol. 403, No. 6, pp. 65-68, 2000. 